\title{
The lethal effect of tryptone-soya broth
}

\author{
PAMELA M. WATERWORTH ${ }^{1}$
}

From the Department of Bacteriology, Royal Postgraduate Medical School, London

SUMMARY Overnight cultures of $V$. cholerae, $V$. el tor, non-cholera vibrio, and Streptococcus pneumoniae in tryptone-soya broth (with $5 \%$ blood added for the last-named) failed to grow on subculture despite visible growth in all tubes. Viable counts and $p \mathrm{H}$ determinations of overnight cultures of various species in tryptone-soya broth, brain-heart infusion broth, and Lab-Lemco peptone broth show that death in tryptone-soya broth is associated with the drop in $p H$ resulting from fermentation of glucose in the medium and that this drop is greater than that produced by the same amount of glucose in brain-heart infusion broth. Some strains of pneumococci failed to grow in brain-heart infusion broth and it is suggested that both these media are unsuitable for blood cultures.

In July 1970 seven strains of $V$. cholerae, eight of $V$. el tor, and five of non-cholera vibrio were received from the late Dr K. Patricia Carpenter; they were on nutrient agar slopes which had been incubated overnight and posted forthwith. On receipt they were subcultured into tryptone-soya broth (the nutrient broth in use in this department at that time): after overnight incubation all tubes were uniformly cloudy but subculture on nutrient agar yielded no growth. Some time before this tubes of tryptone-soya broth with $5 \%$ horse blood were heavily inoculated from freeze-dried cultures of 18 strains of Str. pneumoniae. After overnight incubation all tubes were grossly turbid and Gram films showed large numbers of pneumococci, but 14 failed to grow on subculture.

Tryptone-soya broth contains $0.25 \%$ glucose and it seemed probable that the rapid death of these organisms was due to the drop in $p \mathrm{H}$ due to its fermentation. Dr Carpenter felt that this hazard was not sufficiently appreciated and the following observations are reported at her suggestion: regrettably the work was not completed before her final illness.

\section{Materials and Methods}

Five ml volumes of broth were inoculated with a standard drop of an overnight broth culture. After overnight incubation at $37^{\circ}$ viable counts were ${ }^{1}$ Present address: Department of Microbiology, University College Hospital, Gower Street, London, WC1.

Received for publication 18 November 1971. performed by a modified Miles and Misra technique, six 1 in 10 dilutions being prepared by adding $0.5 \mathrm{ml}$ culture to $4.5 \mathrm{ml}$ peptone water. With eight organisms. $p \mathrm{H}$ was determined at the same time using an EIL pH meter, model 38A.

\section{Medium}

Oxoid no. 2 broth, Oxoid tryptone-soya broth, and Oxoid brain-heart infusion broth (some tests were also done with Difco brain-heart infusion broth). Both tryptone-soya and brain-heart infusion broths contain $0.25 \%$ glucose: a small amount of the former was prepared in this department without glucose.

Viable counts were done on Oxoid no. 2 broth solidified with $1 \cdot 2 \%$ Oxoid no. 3 agar.

Five per cent horse blood was added to all broths and to agar for all strains of streptococci.

\section{Organisms}

With the exception of the Vibrio, all the strains used were fresh clinical isolates.

\section{Results}

The $p \mathrm{H}$ of overnight cultures of eight organisms in Oxoid no. 2 broth, tryptone-soya broth, and brainheart infusion broth and their viable counts are given in Table I. These results show that not only is there a clear relationship between the fall in viable count and drop in $p \mathbf{H}$, but also that the latter is much greater in tryptone-soya broth than brain- 


\begin{tabular}{|c|c|c|c|c|c|c|}
\hline & \multicolumn{2}{|c|}{$L L P$} & \multicolumn{2}{|l|}{$\boldsymbol{B H I}$} & \multicolumn{2}{|l|}{$T S B$} \\
\hline & $\mathrm{p} H$ & Count per $m l$ & $\mathrm{p} H$ & Count per ml & $\mathrm{pH}$ & Count per $m l$ \\
\hline V. cholerae 223 & $6 \cdot 7$ & $750 \times 10^{6}$ & $6 \cdot 0$ & $35 \times 10^{6}$ & $5 \cdot 2$ & Nil \\
\hline Str. pneumoniae 36 & $7 \cdot 1$ & $400 \times 10^{6}$ & No $g$ & & $4 \cdot 8$ & $12 \times 10^{4}$ \\
\hline Str. pneumoniae 935 & $7 \cdot 1$ & $140 \times 10^{6}$ & $6.4^{\circ}$ & $8 \times 10^{6}$ & $4 \cdot 8$ & $1 \times 10^{6}$ \\
\hline Str. pyogenes 479 & $7 \cdot 1$ & $35 \times 10^{6}$ & $6 \cdot 4$ & $90 \times 10^{6}$ & $5 \cdot 8$ & $1 \times 10^{6}$ \\
\hline Str. pyogenes 484 & $7 \cdot 1$ & $15 \times 10^{6}$ & $6 \cdot 6$ & $35 \times 10^{6}$ & 5.9 & $5 \times 10^{6}$ \\
\hline Str. faecalis & $7 \cdot 3$ & $200 \times 10^{6}$ & $6 \cdot 4$ & $1200 \times 10^{6}$ & 5.0 & $1000 \times 10^{8}$ \\
\hline E. coli & $7 \cdot 7$ & $1000 \times 10^{6}$ & $7 \cdot 0$ & $700 \times 10^{6}$ & $6 \cdot 3$ & $3000 \times 10^{6}$ \\
\hline Staph, aureus & $7 \cdot 0$ & $300 \times 10^{6}$ & $6 \cdot 3$ & $450 \times 10^{6}$ & $4 \cdot 7$ & $350 \times 10^{6}$ \\
\hline
\end{tabular}

Table Viable counts and $\mathrm{pH}$ of overnight cultures in three nutrient broths ${ }^{1}$

${ }^{1}$ LLP $=$ Oxoid no. 2 broth, BHI $=$ Oxoid brain-heart infusion broth, TSB $=$ Cxoid tryptone-soya broth

heart-infusion broth, possibly because the former contains less buffering substances.

Counts were done on four strains of Vibrio with similar results in each. The most surprising results were obtained with Str. pneumoniae. All the strains tested grew well in tryptone-soya broth but the overnight count fell in all strains and two cultures yielded no growth from $0.02 \mathrm{ml}$. In contrast six strains failed to grow in brain-heart infusion broth, the medium being unchanged in appearance and $0.02 \mathrm{ml}$ yielding no growth. In the remaining two strains, one yielded only 22 colonies from $0.02 \mathrm{ml}$ and the other is shown in the Table. Four strains which failed to grow were also tested in Difco brain-heart infusion broth; viable counts on these varied from approximately $10 \%$ of that in Oxoid no. 2 broth (one strain) to $0.1 \%$ (two strains) and one strain yielded no growth.

As tryptone-soya agar does not usually contain glucose it seemed possible that this was not essential in the broth. The growth of two strains of $V$. cholerae, one of E. coli, one of Staph. aureus, and six of Str. pneumoniae in tryptone-soya broth without glucose was compared to that in Oxoid no. 2 broth and in every case viable counts were comparable in the two media.

\section{Discussion}

The survival of different species in tryptone-soya broth depends on both their individual susceptibility to acid and also on the extent to which they lower the $p \mathrm{H}$. Thus Str. pyogenes, which survived well in tryptone-soya broth, only reduced the $p \mathrm{H}$ to $5 \cdot 8$, whereas Str. pneumoniae and $V$. cholerae took it considerably lower. Str. faecalis and Staph. aureus are presumably hardier organisms better able to withstand the low $p \mathrm{H}$ they produce.

Tryptone-soya broth was originally adopted in this department because most organisms appeared to grow better in this than in Oxoid no. 2 broth, which had previously been in use. It is clear from the viable counts that the degree of turbidity of an overnight broth culture is not necessarily any guide to its viable content. The Oxoid Manual in its section on tryptone-soya broth states clearly that this medium is not recommended for storing cultures and indeed it must surely be accepted that any medium containing added glucose is unsuitable for this purpose. Glucose broth has specific uses but there can surely seldom be any point in inoculating 'nutrient broth' if the culture is not alive the next morning. Tryptonesoya broth would be a more reliable medium if the glucose were omitted.

No explanation is offered for the failure of pneumococci to grow in brain-heart-infusion broth but the suitability of both this and tryptone-soya broth for blood cultures seems open to question: the latter is no longer in use in this department. 